\title{
Germanium X-Ray Phase Plates for the Production of Circularly Polarized X-Rays*
}

\author{
C.. Yahnke, (i. Srajer, D. Hacffner, D. Mills, L. Assoufid \\ Experimental Facilities Division \\ Advanced Photon Source \\ Argonne National Laboratory \\ Argonne 1160439
}

October, 1993
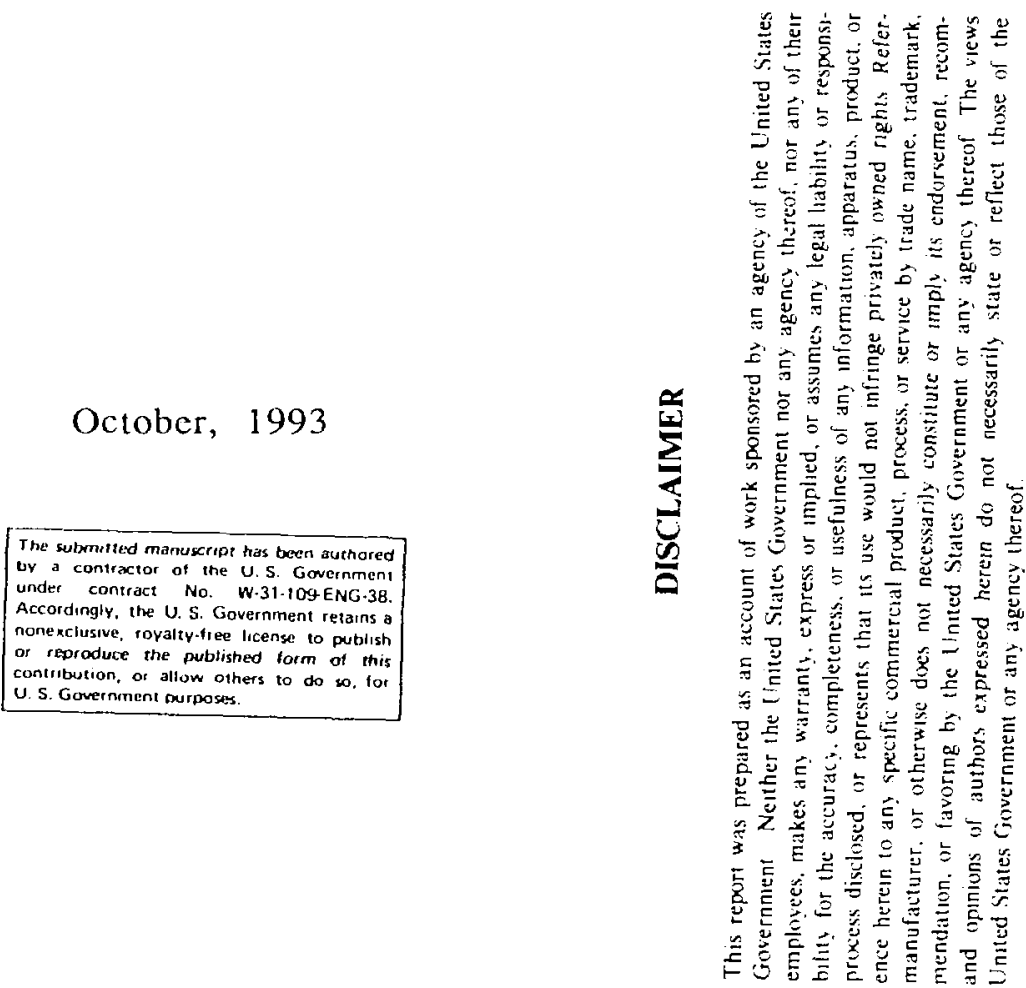

$j w$

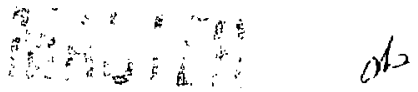

*This work supported by the U.S. Department of Energy, BES-Materials Sciences, under contract no. W-31-109-ENG-38 


\title{
Germanium X-Ray Phase Plates for the Production of Circularly Polarized X-Rays
}

\author{
C. J. Yahnke, (j. Srajer, D. R. Hacifner, D. M. Mills, and L. Assoufid
}

Advanced Photon Source, Argonne National Laboratory, Argonne, IL 60439 USA

We have constructed an $x$-ray phase plate to produce both lincarly and circularly polarized $\mathrm{x}$-rays at discrete energies between $20 \mathrm{keV}$ and $88 \mathrm{keV}$. The plate is a monolithic two-crystal design, constructed from germanium, which increases the resultant degree of circular polarization of the output beam. We have measured the degree of circulatr polarization at $65 \mathrm{keV}$ to be $90 \% \pm$ $4 \%$, significantly better than that produced by silicon phase plates. This radiation was used to measure the magnetic Compton profile for Fe, which was found to be in good agreement with theory and previous work. The underlying $x$-ray optics and the characterization of the device between $62 \mathrm{keV}$ and $93 \mathrm{keV}$ at the Cornell High Energy Synchrotron Source are presented. 


\section{Introduction}

Increases in the available llux of circularly polarized $x$-rays from synchrotron radiation sourees have renewed aterest in their use as a tool with which to probe condensed matter. Many experiments of this nature require sourees with both a high photon flux and a high degree oi circular polarisation. Such soures include off-axis lending magnet radiation, specialized insertion devices, and $x$ ray crystal optics. We have built an $x$-ray optical element, made of single crystal germanium, to produce circularly polarized photons with emphasis in the $50 \mathrm{keV}$ to $1(\mathrm{k}) \mathrm{keV}$ conergy range.

Previous attempts at the use of $x$-ray phase plates for the production of circularly polarized $x$-rays by Golovehenko et al. [1] and Mills [2] focused on the use of silicon perfect crystals as the diffracting element. Their experiments, performed at energies between $10 \mathrm{keV}$ and $55 \mathrm{keV}$, demonstrated that a degree of circular polarization $\left(\mathrm{P}_{\mathrm{c}}\right)$ between $30 \%$ and $75 \%$ could be achieved. At increasing energies, a higher degree of $P_{c}$ can be obtained by using a germanium perfect crystal as the diffracting element rather than silicon. Improvement in the degree of circular polarization is, in our case, motivated by magnetic $x$-ray scattering experiments that are sensitive to the degree of circular polarization of the incident beam $[3,4]$. Additionally, because the cross section for such scattering experiments increases linearly with the incident photon energy [5], the phase plate was designed to operate at energies up to $88 \mathrm{keV}$.

\section{Theory of Operation}

It is well established that a perfect crystal operating in Laue (transmission) geometry is four-refringent at energies that satisfy Bragg's condition [6]. In such instances, there will the four wave fields present in the crystal, each propagating with a distinct wave vector and, therefore, phase velocity. In addition, if one considers that thene are both a diffracted (" $\mathrm{H}$ ") beam and a transmitted (" $\mathrm{O}$ ") beam, then there are actually eight wavefields propagating in the crystal at Bragg's condition. In the following, only the " $\mathrm{H}$ " beam will be used. Therefore, the discussion is limited to the four different wave fields associated with the diffracted beam.

The four wave fields are characterized by both the location of their nodes within the crystal and also the orientation of the incident polarization with respect to the diffracting planes. Those: 


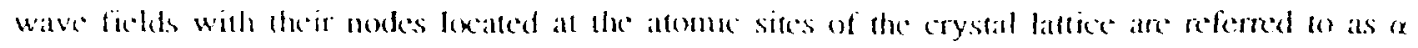

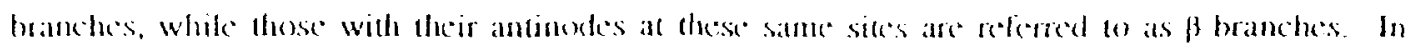

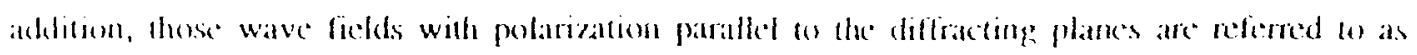
havin!: $\pi$ polatiation, white those wave lields with polariation perpendiculat to the difiateinge plane's ane neferred to as having o polarization. Thus, the four electrie field vectors in the crystal

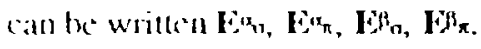

The interaction of the wave felds within the crystal is well described by colovelenko el al. [I] and hant [7]. As already noted, the wave vectors propagating chrough the crystal each propangate: with their own distinct phase velocity, and hence thene is a phase difference between any two given wave vectors at the exit face of the crystal. In the symmetric I aue geometry, this phase difference can be expressed as:

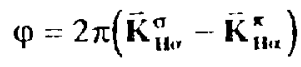

$$
\begin{aligned}
& =\frac{c_{\mathrm{d}}\left|F_{1 I}\right| r_{c} \lambda}{\cos \Theta_{B} a_{0}^{3}}\left(\left(\left(\eta_{\sigma}^{2}+1\right)^{1 / 2}-\eta_{\sigma}\right)-\mid \cos 2 \Theta_{B}\left(\left(\eta_{\kappa}^{2}+1\right)^{1 / 2}-\eta_{\pi}\right)\right),
\end{aligned}
$$

where $h_{1}$ is the thickness of the crystal, $F_{11}$ is the structure factor for the reflection, $\theta_{13}$ is the Bragg angle, $r_{c}$ is the classical electron radius, $\lambda$ is the incident wavelength, a, the lattice spacing, and $\eta_{c, n}$ is a dimensionless quantity proportional to the deviation from the perfect Bragg condition as shown below:

$$
\eta_{\sigma, \pi}=\frac{\pi a_{0}^{3} \Delta \Theta \sin 2 \Theta_{B}}{r_{e} \lambda^{2} P\left|F_{1 I}\right|}
$$

Here, $\Delta \theta$ is the deviation of the incident $x$-ray from the Bragg condicion, and $P=1$ for $\eta_{\sigma}$ and $\cos 2 \theta_{B}$ for $\eta_{\pi}$.

In order for the $x$-rays at the exit face of the erystal to be circularly polarized, two conditions must be: satisfied. First, thene must be a $90^{\circ}$ (or $270^{\circ}, 450^{\circ}$, etc.) phase shift between the two orthogonal polarization states $\sigma$ and $\pi$. Sceond, the amplitude of each of these states must he equal. If these conditions are not satisfied, the resultant beam is elliptically polarized.

Hy chessing the phase difference in equation (1) to be $(2 n+1) \pi / 2 \quad(n=0,1,2, \ldots)$, the first of the two conditions necessary for circular polarization is satistied. This condition determines the set 


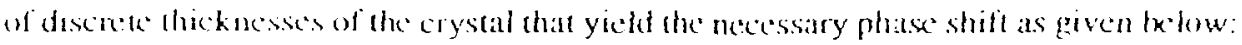

$$
I_{1}=\frac{(2 n+1) \pi a^{3} \cos \theta \theta_{n}}{2\left|r_{1}\right| r_{c} \lambda\left(1-\cos 2 \theta_{0}\right)} \quad\left(\cos \varphi \therefore(2 n+1) \frac{\pi}{2}\right)
$$

To satisfy the second condition necessary for circular polarization, the crystal is rotated by 45" about the incident beam so that its diffracting planes make a $45^{\circ}$ angle with the linearly polaried incident tean from the storage ring. In actuality, the rotation angle is greater than $45^{\prime \prime}$ because, in some cases, the difference in the absorption cofficients between the $\sigma$ and $\pi$ states of the a branch leads to signilicantly different amplitudes at the exit face of the crystal. This is particularly true for lower energies and higher order diffractions.

In Figure 1, a comparison is shown between the performance (at $65 \mathrm{keV}$ ) of a silicon phase plate to one construcked of germanium. From this figure, we can see that, particularly for larger kxan divergences, genmanium clearly outperforms silicon in its ability to polarize the incident $x$ rays. This increased performance is connected to the fact that germanium absorbs more of the $\beta$ branch wave fields than does silicon. These $\beta$ branch wave fields produce circularly polarized $x$ rays of opposite helicity to those produced by the $\alpha$ branch as they are $180^{\circ}$ out of phase with the $\alpha$ branch wave fields. Thus, when combined with the $\alpha$ branch photons in the output beam, the $\beta$ branch plotons reduce its degree of circular polarization.

\section{Construction and Characterization of the Device}

The phase plate is a monolithic, two-crystal design similar to that used by Mills [2] and is shown in Figure 2. The first crystal was cut to the symmetric Bragg geometry, while the second crystal (the phase plate) was cut to the symmetric Laue geometry. The advantages of this combined geometry are the following: first, the doubly diffracted, circularly polarized beam will be parallel to the incident beam. This greatly simplfies the experimental lask of tracking the exit beam as the incident energy is changed. In fact, if the energy is changed by rotating the crystal about its corner (where the latke crystal and the Bragg crystil meet), the exit baim will remain fixed in

space. Second, the incident beam will have its power distributed over a larger area on the Bragge crystal than would be possible if it were incident upon the Laue crystal. Ihis will reduce the heat 
load upen the crystal. The third advantage is the inherent mechanical stability of such a device compared to a separated two crystal device. This advantage is magnitied hy he fact that the I)awin width at these energices is moch kess than an are second. A "weak link," which allows the Bragg crystal to be rotated relatuve to the phase plate, was included to compensilte for the slight difference in the Bragg angle le:tween the Late and Bragg geometry due to index of refraction comections. Alehough this difference is energy dependent, it was of the order of a fraction of an are second in the energy range of our interest. The rotation is perfomed by an electrostrictive actuator (sinilar to a piezolectric tansducer) with a resolution of 0.05 areseconds in our particular geometry.

The (440) diffaction planes were chosen for two reasons. The first reason is practical as the $<110>$ direction is perpendicular to the $\langle$ III $>$ direction, the most readily available boule growth direction. The second reason is that the Bragg angle for the (440) reflection at the highest pessible energies at which the phase plate was designed to work (88 keV). is large enough to permit the corresponding beam footprint to fit on the Bragg crystal.

The step cut in the plase plate allows two different energy ranges to be studied (as shown in 'lable 1). The thickness of each plate was determined from equation (3).

The height of the phase plate and the length of the Bragg crystal are decernined solely from the geometrical considerations arising from the angle of incidence, and to a lesser extent, the size of the incident beam.

Initial charackerization of the phase plate was done by measuring the rocking curve of the crystal, which is the Darwin-Prins curve of the Bragg crystal convoluted with a Laue rocking curve. The experiment was done in-house using the Ka characteristic line ( $E=17.479 \mathrm{keV}$ ) from a sealed molybdenum (Mo) tube operating at $40 \mathrm{kV}$ and $30 \mathrm{~mA}$. Because the width of the perfect Lauc curve at this energy for the (440) planes is 0.41 arc seconds, which is smaller than the Darwin-Prins curve ( $w_{D .1}=1.68$ are seconds) of the Bragg geometry, the measured rocking curve should trace the Darwin-Prins curve. Indeed as shown in Figure 3, the experimental data were in good agrement with the theoretical curve, which, in turn, indicated the crystal boule was of a goud quality and that mounting of the phase plate was relatively strain free.

\section{Description of the Experimental Setup}


The phase plate was lested on the li2 wiggler trambue at the cormell tigh buergy

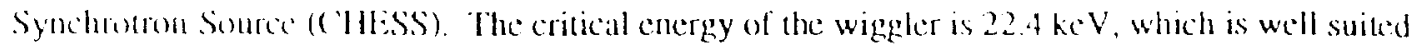
(1) um interest in huh conergy, magnetic Compton scattering experiments.

A lop vicw of the layout of the components in our experiment is shown in figure 4 . A 15 mm-thick carbon filler and a 0.5 -mm-thick copper filter were used lo remowe the lower energy (220) harmonic reflection from the phase plate and also to reduce the effects of heating from the wiggher white bean. This brought the nomal incidence power density at $80 \mathrm{~m} A$ beam current from $15.4 \mathrm{~W} / \mathrm{mm}^{2}$ down to $0.8 \mathrm{~W} / \mathrm{mm}^{2}$. Upon exit from the crystal, the beam passed through a saaled $\mathrm{xe}$ ion chamber, which acted as a beam monitor, before its polarization was measured by means of a compten polarimeter similar to that used by Snend et al [8] and Schildkamp [9]. While this type of polarimekr can measure the intensity of the orthogonal elecuric fields, it cannot measure the relative plyse difference between the two components. Thus, the polarineter can measure the degrec of linear polarization, but not the degree of circular polarization. This measurement is used to determine the energies for which the circular polarization is a maximum by idencifying the energies for which the lincar polarization is a minimum $\left(P_{L}=0\right)$. The degree of circular polarization $\left(\mathrm{P}_{c}\right)$ at these energies is then measured by a magnelic Compton-scattering experiment.

The magnetic Compton-scattering experiment required the removal of the polarimeter and the installation of an electromagnet with an Fe target placed hetween the poles. The Compton scattered photons were collected using a Ge solid-state detector, and their energies were resolved using a multichannel analyaer.

\section{Lincar Polarization Measurement}

The polarimeter used Nal scintilation counters placed at $90^{\circ}$ relative both to each other and the direction of the incident beam to measure the Compton scattered radiation from a $1-\mathrm{cm}$-thick aluminum target. This design differed from that of Smend and Schildkamp in two ways. First, the target was aluminum, not kapton or beryllium, to provide a significant amount of scattering at high photon energies. Second, both detectors viewed the sample in a reflection-reflection geometry rather than in a transmission-reflection geometry. This insures that eadh detector sees an 


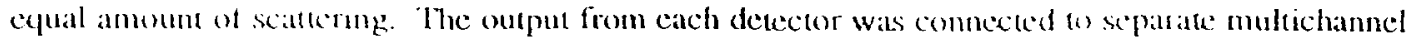
amalyers. The melastic or compton peak cornesponding to the correct ine ryy wats integrated over the region of interest (ROH) for each detector. The ROI was contered upon the peak of the Comptom protile and was delined to include the \pm 10 a.u. of momentum. This gave two quantities: one that was proportional to the degree of linear polarization parallel (h) to the storage ring plane of orbit (detined to be the horiental plane) and another that was proportional to the degree of linear polarization perpindicular to this plane $(1$,$) . The degree 10$ which the knam was linearly polarized $\left(P_{1}\right)$ was calculated as follows:

$$
P_{t}=\frac{I_{11}-I_{1}}{I_{11}+I_{1}}
$$

lirom this definitom, it follows that $P_{\mathrm{L}}=-1(+1)$ comesponds to $100 \%$ polariotion in the vertical (horizontal) plane. To reduce contamination in the Nal detectors from fluorescence, 1/8"'-thick lead disks with a 1.5-mu pinhole were placed in front of each detector. To minimize systematic errors that could have arisen from the slight difference in the size of the pinholes, two sets of data were collected for each energy by rotating the Compton polarimeter by $90^{\circ}$ about the exit beam from the phase plake.

The above procedure yicks $P_{1}$ for a particular energy. Equation 1 shows that, as the incident Bragg angle is changed (and hence the incident energy upon the phase plate), the phase hetween $E^{*}{ }^{\prime}$ and $E \omega_{\pi}$ changes as well. This will, in turn, affect the degree of lincar and circular polarization. Thus, $\mathrm{P}_{\mathrm{l}}$, was measured as a function of energy over the range $62 \mathrm{keV}$ to $93 \mathrm{keV}$. The result is shown in Figure 5. From this figure, one can see the linear polarization change from horizontal linear ( $1:>88 \mathrm{keV}$ ) to vertical linear (at $75 \mathrm{keV}$ ) and back to horizontal linear $(\mathrm{E}<65$ $\mathrm{keV}$ ), where this cycle will continue to repeat itself as the energy is decreased. The energies for which $\mathrm{P}_{\mathrm{L}}=0$ are cither $45^{\circ}$ linearly polarized, circukarly polarized, or unpolarized. To climinate the possibility of $45^{\circ}$ lincar polarization, $\mathrm{P}_{\mathrm{L}}$ was measured after the Compton polarimeter was rotated by $45^{\circ}$ about the incident beam. No change in $P_{1}$ was measured, so the bean could not have been $45^{\circ}$ lincarly polarized.

From Figure 5, we can clearly see the points at which the linear polarization is a minimum, 
and the agreement with theory is good in this region. At these points ( $88 \mathrm{keV}$ and $65 \mathrm{keV}$ ), thi* degree of circular polarization is at a maximum. In the regions for which the exit beam is highly linearly polarized $(7.5 \mathrm{keV})$, multiple scatering effects within the target aceount for the discrepancy between the data and theory. No corrections for multiple scattering have been made though as our interest in the precise value of $P_{L}$ is only for $P_{L}=0$.

\section{Circular Polarization Measurement}

In order to confirm that the exit beam at $65 \mathrm{keV}$ and $88 \mathrm{keV}$ was indeed circularly polarized (and not unpolarized), a magnetic Compton-scaltering experiment was performed using a polycrystalline iron target. Two sets of adta wene separately collected for opposite directions of the applied magnetic ficld in an ABBA sequence using a multichannel analyzer. One $A$ or $B$ step was approximately $5 \mathrm{~min}$. long. Data were collected at a ratc of 262 counts/s (in the Compton peak) for four hours. The profiles for opposite directions of the magnetic field were subtracted and these results were then summed for all of the sequences to produce a single magnetic Compton profile (MCP). The integral of the profile from -10 a.u. to +10 a.u. is nomalized to the number of magnetic elcctrons/atom (2.2 for Fe) to yield the profile shown in Figure 6. These data are in good agreement with that obcained by Mills \{4] and Cooper et al. [3] and the theory calculated by Wakoh

and Kubo [10]. From this profile in Figure 6, the degree of circular polarization $P_{c}$ at $65 \mathrm{keV}$ was calculated to be $90 \% \pm 4 \%$. The exact value of $P_{c}$ at $88 \mathrm{keV}$ could not be calculated due to contamination of the Compton profile by lead fluorescence $\left(K \alpha_{1}, K \alpha_{2}\right.$, and $\left.K \beta\right)$.

\section{Summary of Results}

We have shown that a germanium phase plate can be used to convert linearly polarized radiation to circularly polarized radiation at high $\mathrm{x}$-ray energies (65 keV and $88 \mathrm{keV}$ ). The degree of this circular polarization has been shown to be $90 \% \pm 4 \%$, significantly better than that produced by silicon phase plates. This radiation was used to measure the magnetic Compton 
profike of Fe, which was found to be in good agsecment with theory and previous work.

\section{Acknowledgements}

The authors would like to thank Prof. N. Sakai and the staff members of both the CHESS and Experimental tacilities Division at the APS for valuable discussions. This work is supported by the U.S. D)( LE-13LS under Contract No. W-31-109-ENG-38.

\section{References}

[1] J.A. Golovchenko, B.M. Kincaid, R.A. Lovesque, A.E. Meixner and D.R. Kaplan, Phys. Rey. Letl. 57 (1986) 202.

[2] D.M. Mills, Nucl. Inst. and Meth. A 266 (1988) 531.

[3] M.J. Cooper, D. Laundry, D.A. Candwell, D.N. Timms, R.S. Holt, and G. Clark, Phys. Rev. B 34 (1986) 5984.

[4] D.M. Mills, Phys. Rev. B 36 (1987) 6178.

[5] M. Blume, J. Appl. Phys. 57 (1985) 3615.

[6] B.W. Batterman and H. Cole, Rev. Mod. Phys. 36 (1964) 581 .

[7] M. Hart, Philosophical Magazine B 38 (1978) 41.

[8] F. Smend, D. Schaupp, H. Czerwinski, A.H. Millhouse and H. Schenk-Strauss, DESY SR-84-003 (1984).

[9] W. Schildkamp, private communication.

[10] S.Wakoh and Y. Kubo, J. Magn. Mater. 5 (1977) 202. 


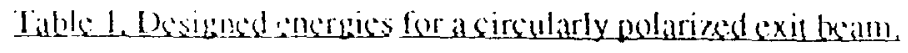

\begin{tabular}{|c|c|c|}
\hline Plabs: (') & $\begin{array}{c}\text { Energy for thin } \\
\text { pidt }(k+v)\end{array}$ & $\begin{array}{c}\text { Energy for thick } \\
\text { pun (kiv) }\end{array}$ \\
\hline$y(1$ & 40.880 & 88.457 \\
\hline 270 & 27.536 & 6.5 .427 \\
\hline 4.50 & 22.829 & 55.217 \\
\hline 030 & 20.147 & 49385 \\
\hline
\end{tabular}

Figure 1. (omparison of the total degree of circular potarization for both gemanium (solid line) and silicon (broken line) as a function of input beam divergence. Note that, even for large beam divergences, the germanium still has a polarization of approximately $86 \%$-searly twice that of silicon.

Figure 2. Photograph of the phase plate. The length, height, and width of the crystal are $120 \mathrm{~mm}$, $35 \mathrm{~mm}$ and $35 \mathrm{~mm}$ respectively.

Figure 3. Comparison of the rocking curve data (open circles) taken at $17.479 \mathrm{keV}$ (Mo Ka) with theory (solid line). The theory was determined by convoluting a Laue rocking curve ( 0.41 are seconds) with the Darwin-Prins curve ( $\mathrm{W}_{\mathrm{D}-\mathrm{P}}=1.68$ are seconds). The Darwin width of this convoluted curve is 1.98 are seconds.

Figure 4. Schematic top view of setup used. The incident white beam is linearly polarized in the plane of this page. The srystal is rotated by $45^{\circ}$ about the incident beam to produce a monochromatic, circularly polarized exit heam. The Compton polarimeter is removed when the magnetic Compton scattering experiment is performed.

Figure 5. Comparison of the measured degree of lincar polarization (\%) with theory. No corrections for multiple scattering were made. The data are shown for two different positions of the Compton polarimeter. Position 2 (open diamond) is that in which the polarimeter is rotated by $90^{\circ}$ from position 1 (open circle) about the exit beam from the phase plate.

Figure 6. Magnetic Compton profile (MCP) for Fe at $65 \mathrm{kcV}$ incident photon energy. The agreement between the data (solid circle) and theory (broken line) [10] are good. Data from both Mills (open sejuare) [4] and Cooper et al. (open triangle) [3] are presented for comparison. The lack of a null result indicates that the incident radiation is circularly polarized. The integral of this profike determines the degree of circular polarizaion. 
Degree of Circular Polarization

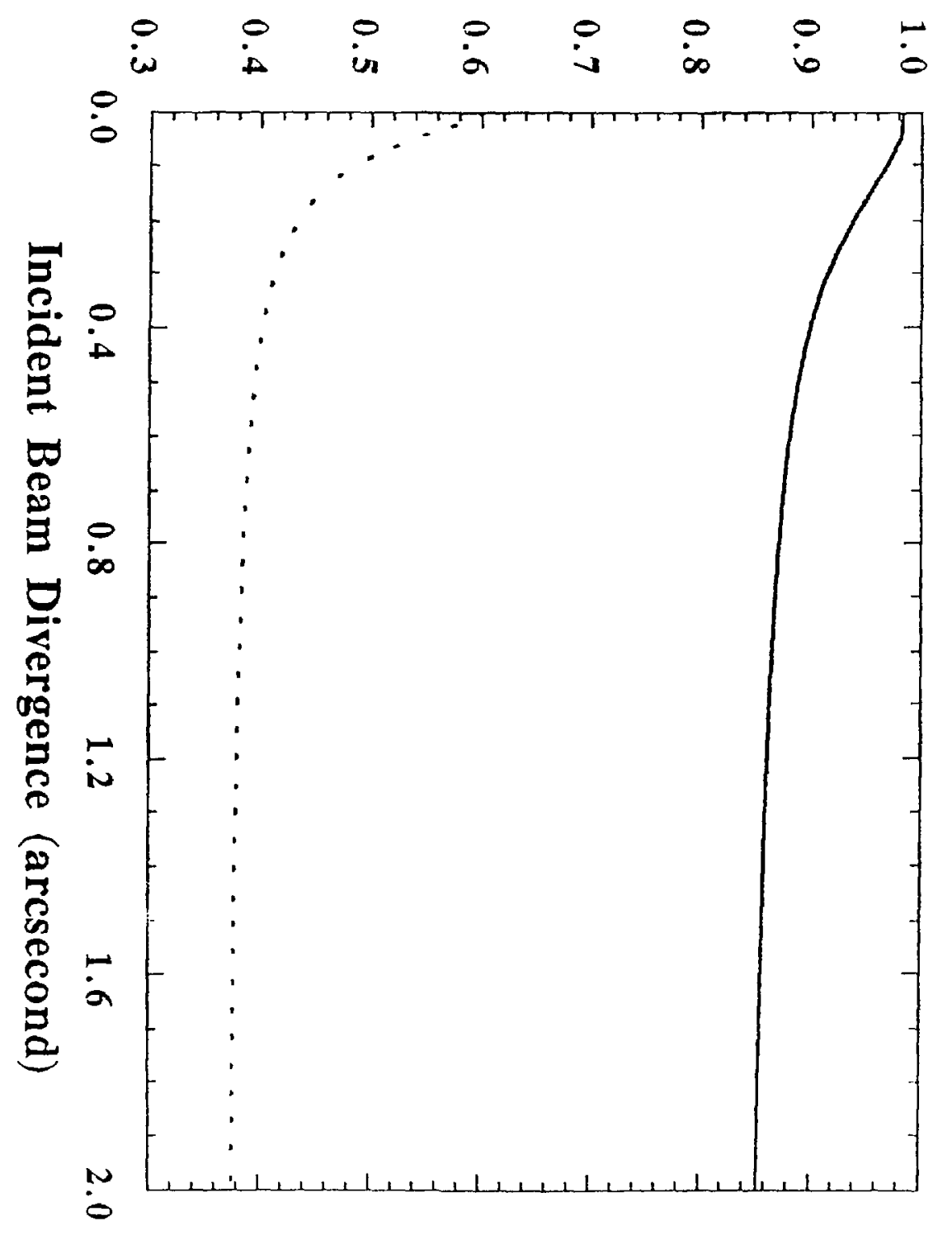




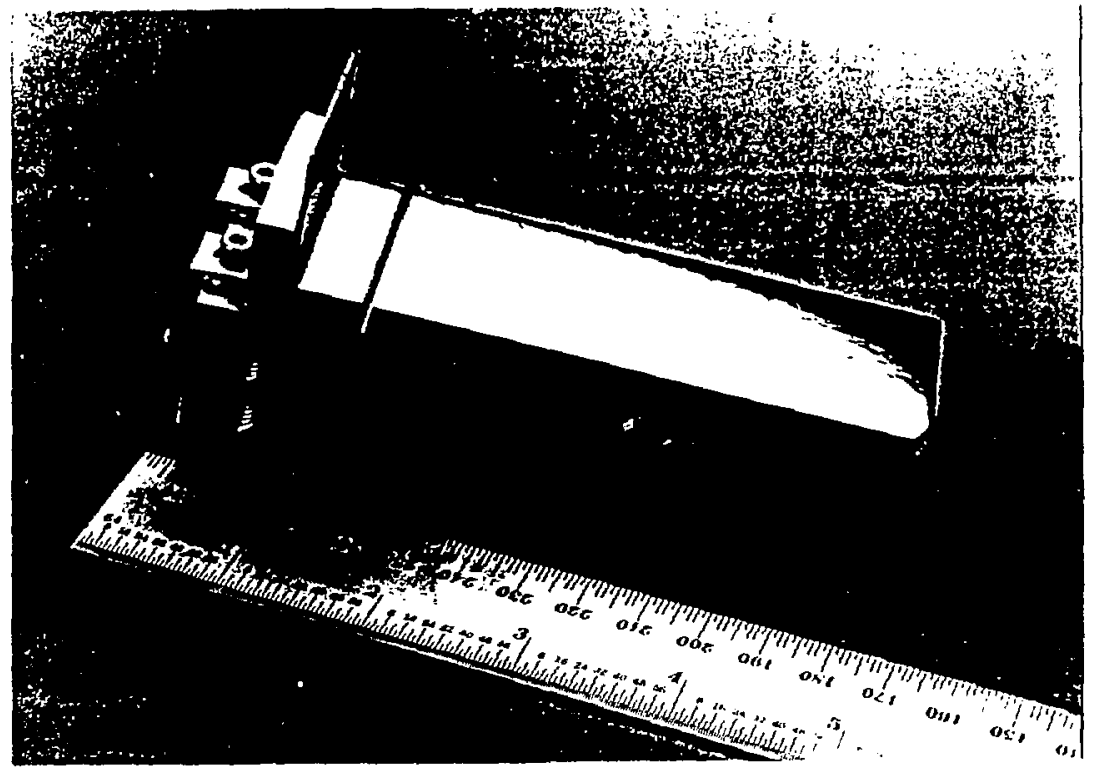




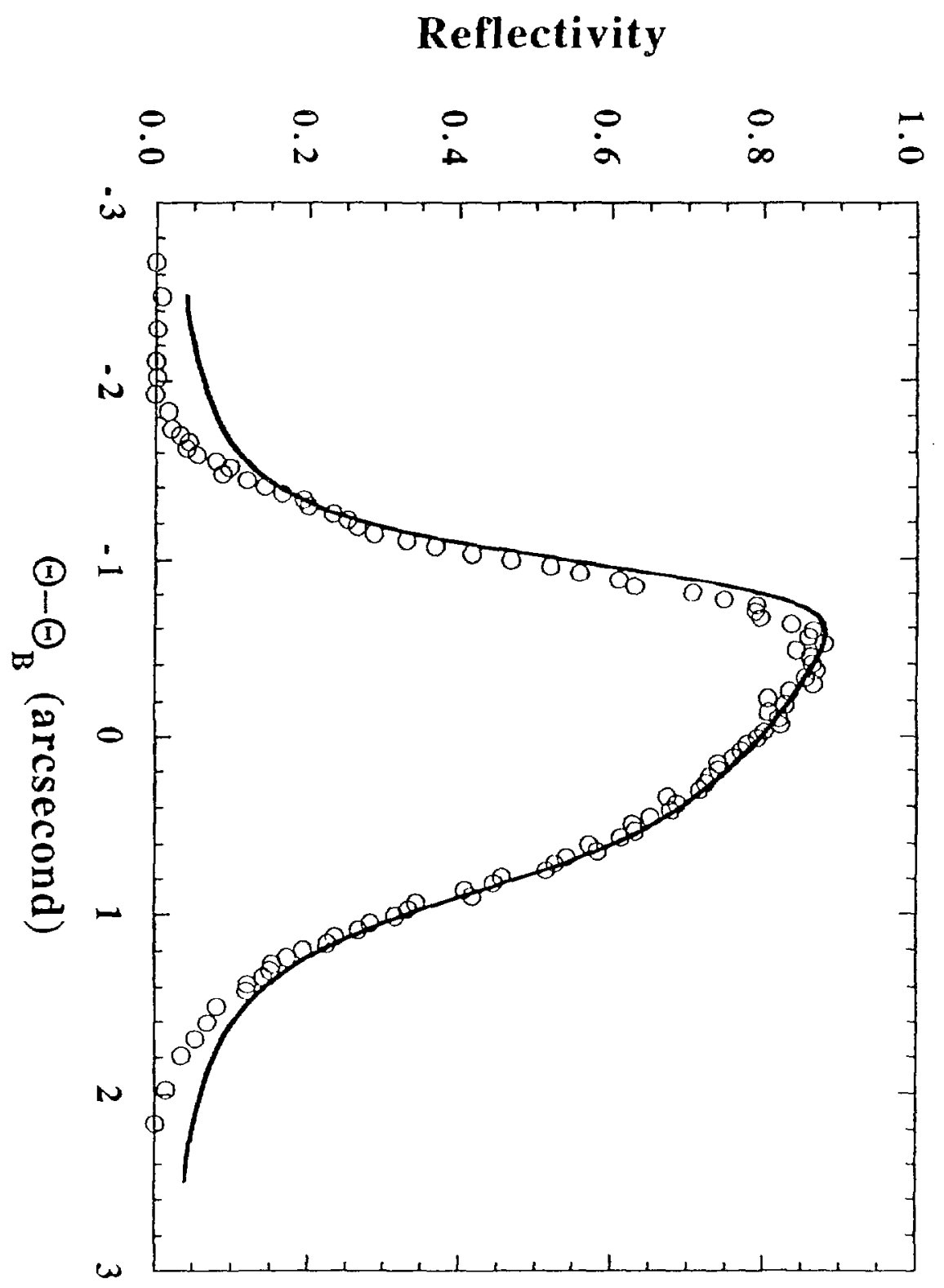


Degree of Linear Polarization (\%)

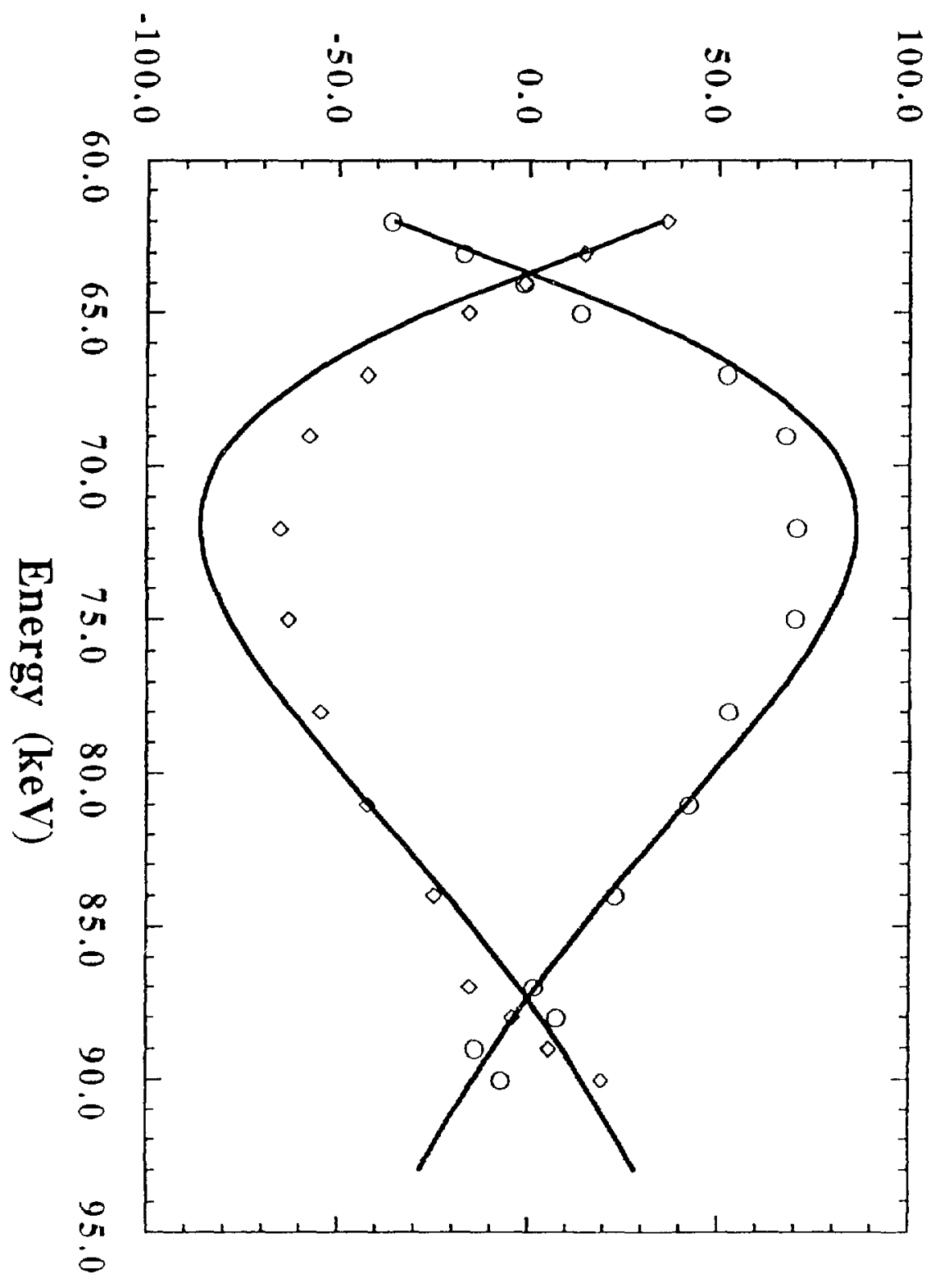




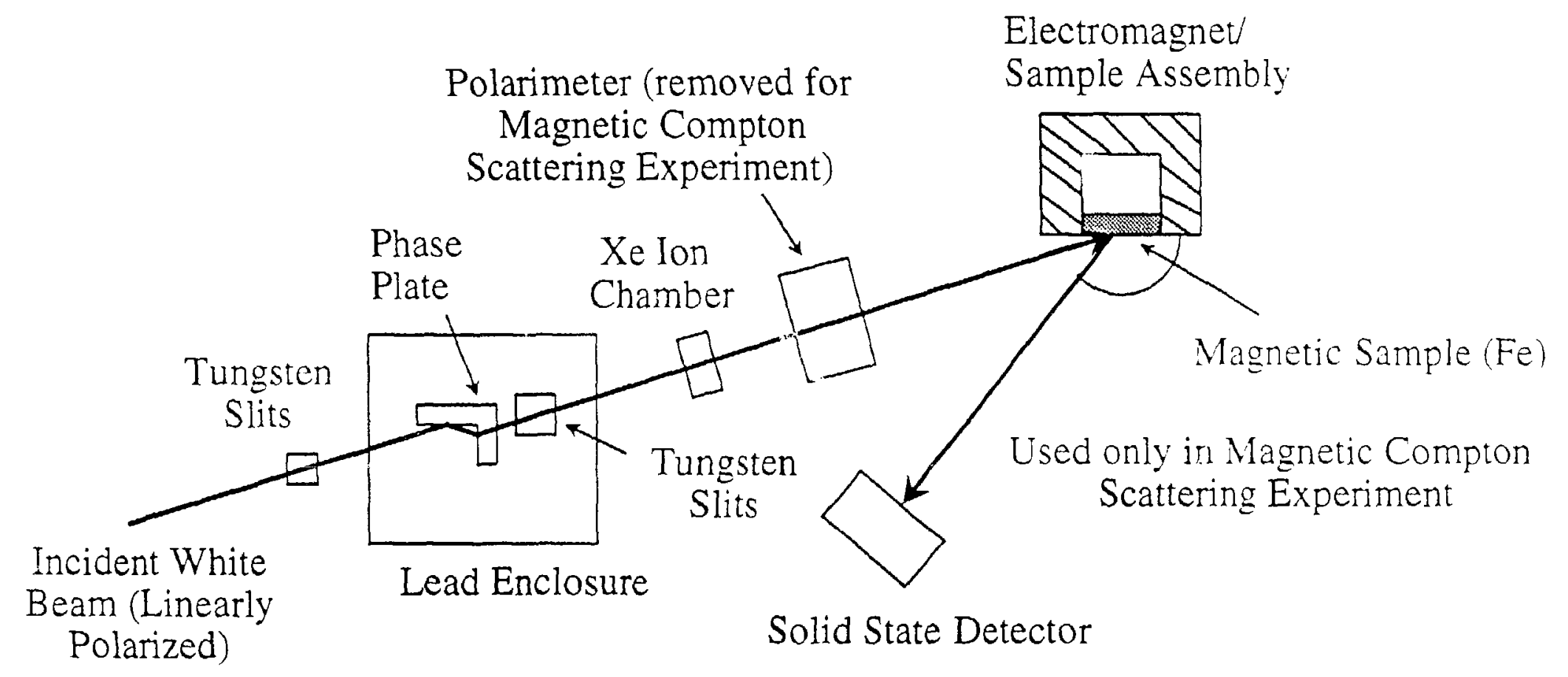




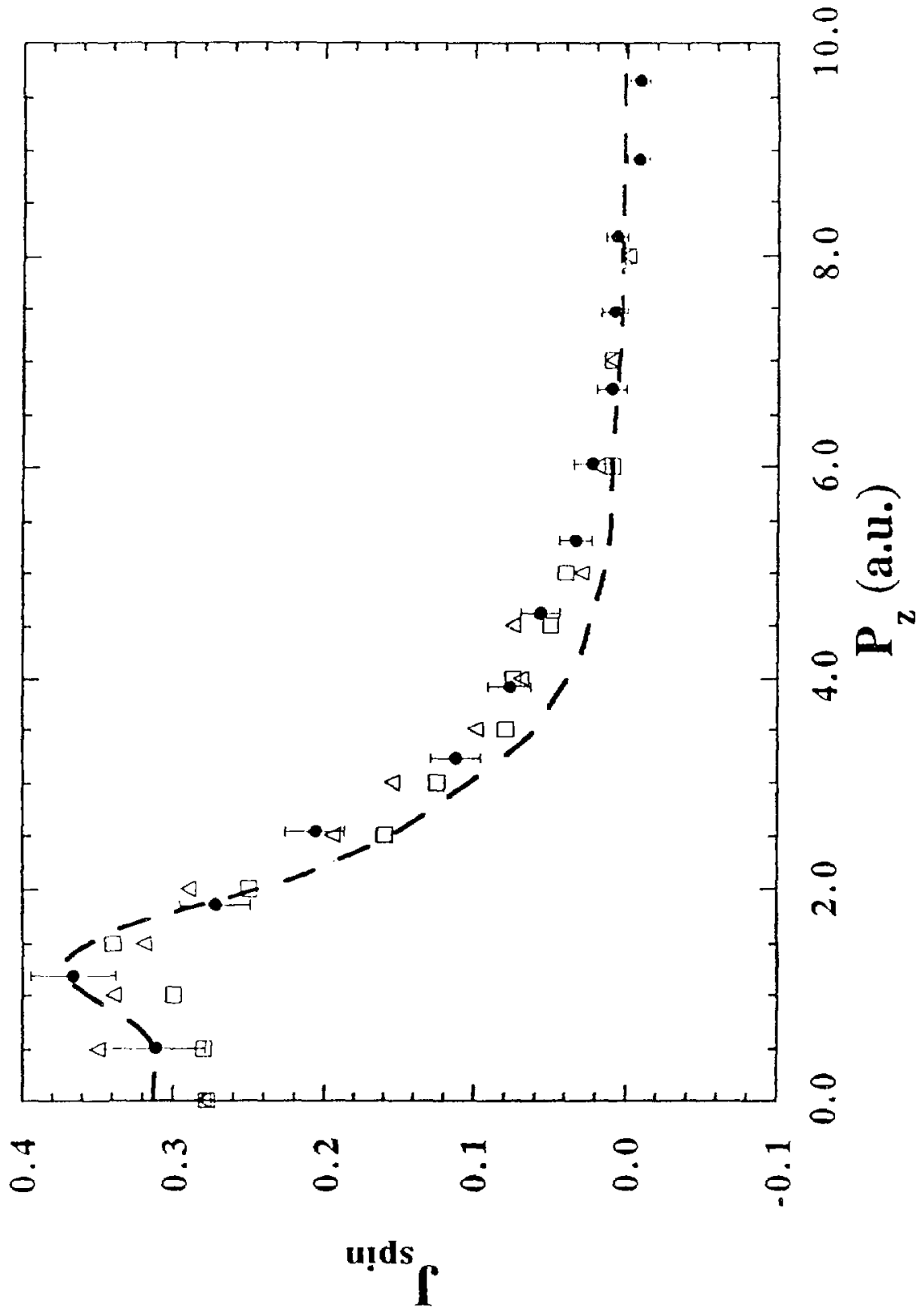

